\title{
Arm reduced robotic-assisted laparoscopic hysterectomy with transvaginal cuff closure
}

\author{
Serkan Bodur ${ }^{1}$, Murat Dede ${ }^{1}$, Ulas Fidan ${ }^{1}$, Burcin F. Firatligil ${ }^{1}$, Mustafa Ulubay ${ }^{1}$, Mustafa Ozturk², Mufit C. Yenen ${ }^{1}$ \\ ${ }^{1}$ Department of Gynecology and Obstetrics, University of Health Sciences, Gulhane Training and Research Hospital, Ankara, Turkey \\ ${ }^{2}$ Department of Gynecology and Obstetrics, University of Health Sciences, Bakirkoy Dr. Sadi Konuk Training and Research Hospital, \\ Istanbul, Turkey
}

Videosurgery Miniinv 2017; 12 (3): 271-276

DOI: https://doi.org/10.5114/wiitm.2017.68772

\begin{abstract}
Introduction: The use of robotics for benign etiology in gynecology has not proven to be more beneficial when compared to traditional laparoscopy. The major concern regarding robotic hysterectomy stems from its high cost.

Aim: To evaluate the clinical utility and effectiveness of one-arm reduced robotic-assisted laparoscopic hysterectomy as a cost-effective surgical option for total robotic hysterectomy.

Material and methods: A sample population of 54 women who underwent robotic-assisted laparoscopic surgery for benign gynecologic indications was evaluated, and two groups were identified: (1) the two-armed robotic-assisted laparoscopic surgery group ( $n=38$ patients), and (2) the three-armed robotic-assisted laparoscopic surgery group ( $n=16$ patients).

Results: An increased cost was observed when three-armed robotic surgery was employed for benign gynecologic surgery ( $p$ 0.001). The cost reduction observed in the study group was primarily derived from one robotic arm reduction and vaginal closure of the cuff. This cost reduction was achieved without an increase in complication rates or undesirable postoperative outcomes. An estimated profit between $\$ 399.5$ and $\$ 421.5$ was made for each patient depending on the suture material chosen for cuff closure. Two-armed surgery resulted in an 18.6\% reduction in procedure-specific costs for robotic hysterectomy.

Conclusions: Two-armed robotic-assisted laparoscopic hysterectomy appears to be a cost-effective solution for robotic gynecologic surgery. This surgical solution can be performed as effectively as classical three-armed robotic hysterectomies for benign indications without the risk of increased surgical-related morbidities. This approach has the potential to be a widely preferred surgical approach in medical communities where cost reduction is one of the primary determinants of surgery type.
\end{abstract}

Key words: hysterectomy, total robotic hysterectomy, vaginal cuff closure.

\section{Introduction}

Hysterectomy is the most common gynecologic surgical procedure performed worldwide. Each year, approximately 600,000 hysterectomies are performed in the United States (U.S.) alone, accounting for a total annual expenditure of approximately
$\$ 5$ billion [1]. Minimally invasive surgical techniques are increasingly used in the practice of hysterectomy $[2,3]$. While laparoscopic hysterectomies accounted for only $14 \%$ of all benign hysterectomies in 2005 , this figure rose to $30.5 \%$ in only 5 years in the U.S. $[4,5]$. The acceptance as well as the prevalence of robotic hysterectomy increased more rapidly than for

\section{Address for correspondence}

Serkan Bodur MD, Department of Gynecology and Obstetrics, University of Health Sciences, Gulhane Training and Research Hospital,

General Tevfik Saglam Street, 06010 Ankara, Turkey, phone: +90 5336379313, fax: +90 3123045800, e-mail: serkan_bodur@yahoo.com 
any other minimally invasive method, accounting for $9.5 \%$ of all hysterectomies by 2010, a mere 5 years after its approval by the U.S. Food and Drug Administration in 2005 [2-4, 6].

The major advantage of robotic surgery over open surgery and laparoscopy is improved mastery due to enhanced movements achieved through the unique articulations of the robotic arms, which operate almost completely absent of tremor, coupled with better ergonomic position for the surgeon afforded by the console of the surgery unit $[7,8]$. Even though it has not been corroborated in the field of gynecology, robotic surgery is believed to be associated with decreases in blood loss, postoperative pain, use of pain medications and length of hospital stay $[2,9]$. Although there is a wide array of studies based on single institution data, the use of robotics for benign etiology in gynecology has not proven to be more beneficial than traditional laparoscopy according to reports of randomized controlled trials (RCTs) [10-13]. The major concern regarding robotic hysterectomy stems from its high cost [6, 14-18]. In general, the cost of robotic hysterectomy was found to be $16 \%$ to $34 \%$ higher than that of laparoscopic hysterectomy $[14-16,18]$. Additionally, hospital costs per case of robotic hysterectomy were found to be approximately $\$ 2,489$ higher than for laparoscopic hysterectomy [14].

\section{Aim}

In our center, one-arm reduced robotic assisted laparoscopic hysterectomy was used due to the urgent need to reduce expenditures of robotic gynecologic surgery. In this study, we strive to analyze and demonstrate the feasibility of the reduction of an arm during robotic hysterectomy and its attendant effects on surgical costs and outcomes. The reduction of an arm in direct terms means avoidance of a needle driver, together with draping of the arm, barbed suture for vaginal vault closure, and a transvaginal approach for vaginal cuff closure.

\section{Material and methods}

\section{Patients and data collection}

A retrospective case-control study was undertaken. Data pertaining to all robotic hysterectomies performed at Gulhane Training and Research Hospital from January 2011 to December 2016 were obtained from both electronic medical records and clinical case records. Only data for hysterectomies performed with benign indications were collected for the study. All procedures were performed by certified robotic surgeons and fellows. Information gathered from the patient charts included age, body mass index (BMI), prior abdominal surgery including cesarean section, preoperative and postoperative diagnosis based on pathology reports, length of hospitalization, perioperative hemoglobin changes, operative time, conversion to open surgery, blood transfusions, intraoperative complications - including ureteral, vascular and intestinal - and other injuries. Operative time was defined as the time between the start and the finish of the procedure according to operating room records. Surgical outcomes of robotic hysterectomies performed using two arms and three arms were compared. The study was reviewed and approved by the Gulhane Military Medical Academy review board.

\section{Surgical technique}

The da Vinci Si Surgical System (Intuitive Surgical, Inc., Sunnyvale, CA, U.S.) was used to perform the robotic hysterectomies. The patient was placed in the neutral position, and a pneumoperitoneum was established with a Veress needle. A $12 \mathrm{~mm}$ trocar, to be used for the camera arm, was placed at the umbilicus or higher in the midline abdomen depending on the size of the uterus. The patient was then placed under general anesthesia in the Trendelenburg position. The left and right $8 \mathrm{~mm}$ trocars for robotic arms ((8 $\mathrm{mm}$ disposable bladeless obturator) (Intuitive Surgical, Inc., Sunny-vale, CA, USA)) were placed 8 to $10 \mathrm{~cm}$ lateral and 2 to $3 \mathrm{~cm}$ inferior to the camera, as if they were creating an arch around the uterus. A $10 \mathrm{~mm}$ assistant port was placed into the left upper quadrant, 5 to $7 \mathrm{~cm}$ superior to and midway between the camera port and the left upper robotic trocar. After trocar placement, the da Vinci robot was docked, and instruments were introduced under direct visualization: Hot Shears (Monopolar Curved Scissors, Intuitive Surgical, Inc., Sunnyvale, CA, U.S.), fenestrated bipolar forceps (Intuitive Surgical, Inc., Sunnyvale, CA, U.S.) and, if needed, a large needle driver (Intuitive Surgical, Inc., Sunnyvale, CA, U.S.). A V-Care uterine manipulator (ConMed Corporation, Utica, NY, U.S.) was placed in all hysterectomies. Colpotomy was performed using a monopolar 
coagulation current (50 W) through a well-fitted colpotomy ring. Vaginal cuff closure with intracorporeal suturing was performed in a single-layer fashion either by non-locking running sutures of polyglactin (Vicryl; Novartis, Basel, Switzerland) on a CT-1 needle followed by interrupted figure of eight sutures or by using barbed suture closure with V-Loc 180 unidirectional polyglyconate monofilament (Covidien, Mansfield, MA, U.S.) barbed sutures. The vaginal cuff was closed in full thickness fashion by incorporating the vaginal epithelium and the pubocervical fascia in each bite. Transvaginal vaginal cuff closure was performed with a two-layer technique using braided and coated 0 polyglactin suture on a half circle HR26 needle.

\section{Statistical analysis}

Collected data were analyzed by Statistical Package for Social Sciences version 14.0 (SPSS Inc., Chicago, IL, U.S.). Continuous variables were expressed as mean \pm standard deviation, whereas categorical variables were denoted as numbers or percentages where appropriate. The Smirnov-Kolmogorov test was used to test the distribution of data. Associations were tested using $\chi^{2}$ for categorical variables and Student's $t$ test for continuous variables where appropriate. A $p$-value of less than 0.05 was considered statistically significant.

\section{Results}

The demographic variables and clinical characteristics of the two surgery groups are shown in Table I. Only parity was found to be statistically significant $(p=0.010)$. The comparison of hospitalization time (in days) and operation time (in minutes) was found to be statistically significant between the two groups at $p \leq 0.001$ for each (Table II). None of the two-armed procedures were converted to threearmed hysterectomy.

Table I. Patient demographics $(N=54)$

\begin{tabular}{|lccc|}
\hline Parameter & Two operative armed TRH $(n=38)$ & Three operative armed TRH $(n=16)$ & $P$-value \\
\hline Age $[$ years] & $50.00 \pm 5.46$ & $47.87 \pm 3.81$ & $0.163^{*}$ \\
\hline BMI $\left[\mathrm{kg} / \mathrm{m}^{2}\right]$ & $29.35 \pm 4.44$ & $26.71 \pm 3.55$ & $0.207^{\star}$ \\
\hline Gravida & $2.60 \pm 1.24$ & $3.18 \pm 1.16$ & $0.116^{\star}$ \\
\hline Parity & $1.68 \pm 0.70$ & $2.18 \pm 0.40$ & $0.010^{*}$ \\
\hline Prior surgery (\%) & 44.7 & 31.2 & $0.367^{* *}$ \\
\hline \begin{tabular}{l} 
Indications (\%): \\
\hline Leiomyoma
\end{tabular} & $14 / 63.6$ & & $0.228^{* *}$ \\
\hline Abnormal bleeding & $9 / 64.3$ & $5 / 36.3$ & \\
\hline Others & $15 / 83.0$ & $3 / 35.7$ & \\
\hline
\end{tabular}

${ }^{*}$ Student's t-test was used, ${ }^{* *} \chi^{2}$ test was used.

Table II. Surgical outcomes

\begin{tabular}{|lccc|}
\hline Parameter & Two operative armed TRH $(n=38)$ & Three operative armed TRH $(n=16)$ & $P$-value* \\
\hline Operative time $[\mathrm{min}]$ & $103.42 \pm 39.06$ & $175.31 \pm 31.80$ & $<0.001$ \\
\hline Hospital stay & $3.00 \pm 0.69$ & $4.75 \pm 2.01$ & $<0.001$ \\
\hline Perioperative hemoglobin change & $4.86 \pm 4.22$ & $4.22 \pm 2.98$ & 0.689 \\
\hline Conversion to laparotomy & 1 & 1 & 2 \\
\hline Transfusion & 2 & 1 & \\
\hline Any organ injury & 0 & 0 & \\
\hline Vaginal cuff dehiscence & 0 & \multicolumn{2}{c|}{} \\
\hline
\end{tabular}


Table III. Procedure specific cost differences between two- and three-port robotic hysterectomy

\begin{tabular}{|lc|}
\hline Procedure & Cost [\$] \\
\hline Large needle holder & 352 \\
\hline 8 mm disposable bladeless obturator & 40 \\
\hline Instrument arm drape & 7.5 \\
\hline Total & 399.5 \\
\hline V-Loc barbed suture* & 22 \\
\hline Total cost together with V-Loc & 421.5 \\
\hline
\end{tabular}

*When preferred.

\section{Cost measurements}

The procedure-specific robotic hysterectomy cost was calculated by taking into account the cost of equipment, specifically the actual number of robotic instruments, drapes and barbed sutures in cases where they were used for vaginal cuff closure. The other direct costs, such as operation room cost, robot amortization and periodic maintenance costs, were all ignored together with indirect costs derived from day of hospital stay, nursing, recovery room and other facilities. The procedure-specific robotic hysterectomy cost of two- and three-armed robotic hysterectomy was $\$ 1,739$ and $\$ 2,140.50$ per case, respectively. The procedure-specific cost difference between the two- and three-armed robotic hysterectomy groups was primarily attributed to the use of the large needle holder, one additional $8 \mathrm{~mm}$ disposable bladeless obturator, and the instrument arm drape, which cost \$352, \$40 and $\$ 7.50$ per case, respectively. The total cost difference between two- and three-armed robotic hysterectomy was $\$ 399.50$ dollars per case (Table III).

\section{Discussion}

The present study sought to analyze the feasibility and effectiveness of one arm reduction in robotic hysterectomy. Intraoperative complication rates did not differ between the two approaches (two-armed vs. three-armed) in robotic hysterectomy. None of the one-arm reduced cases were converted to classical three-armed robotic hysterectomy. Additionally, we could not distinguish any difference between the two approaches in terms of surgical outcomes.

Because surgical outcomes using the two approaches appear very similar, benefits gained through the arm reduction have become increasingly import- ant to both the surgeon and the patient. The primary benefit demonstrated by this study was a $\$ 399.50$ cost reduction achieved for each surgical case in which a two-armed robot was used. The RCTs conducted to assess the effectiveness of robotics in benign gynecologic surgery did not demonstrate any benefit [10-13]. Additionally, studies conducted to analyze the cost-effectiveness of robotic surgery revealed that it was the most expensive method for hysterectomy, although the open abdominal method resulted in longer length of stay and longer operation times when compared to robotic surgery [19, 20]. We contend that the cost benefit gained by the reduction of an arm may be very important, especially in lower-income countries. The $18.6 \%$ reduction in the procedure-specific costs of two-armed robotic hysterectomy may well make it more cost-effective than traditional three-armed robotic hysterectomy, even for benign gynecologic surgery.

The reduction of the needle holder arm rendered transvaginal closure of the vaginal cuff mandatory for accomplishment of the surgery. Recently, vaginal cuff dehiscence has been recognized as one of the additional drawbacks of robotic hysterectomy, with closure of the vaginal vault through the robotic route by intra-corporal suturing shown to be related to a nearly nine-fold increase in the risk of vaginal cuff dehiscence [21]. Previous studies that have assessed the incidence and patient characteristics of vaginal cuff dehiscence following robotic surgery determined the dehiscence rate to be $4.1 \%$ and raised concerns that the vaginal closure technique may be a major contributing factor to this complication [22]. Furthermore, a recent large cohort study suggested that transvaginal colporraphy is associated with a nine-fold reduction in the risk of vaginal cuff dehiscence when compared to robotic suturation of the vaginal cuff. Although vaginal cuff dehiscence occurs in only one of 300 instances of total hysterectomy, we did not encounter any dehiscence in those cases outlined herein following transvaginal closure of the cuff during robotic hysterectomy [8, 21, 22]. Based on relevant extant literature, mandatory transvaginal closure of the vaginal cuff might be considered a secondary gain derived from the two-armed robotic approach, in addition to the previously identified benefit of cost reduction. Patients may also obtain additional cosmetic or esthetic benefits because one less abdominal puncture is required during the twoarmed robotic procedure. 
This study has a few weaknesses. A relatively small patient participation rate or, in other words, an insufficient sample size was one of the important weaknesses of the study that prevented us from reaching a more definitive conclusion. Retrospective data retrieval and the relatively long duration of the study represent additional aspects of the study that merit examination and potential criticism. Although the data retrieved from the patient charts were relatively objective, they were not uniform, having been recorded by various staff members over the course of time. A third bias that also represents a strength of the study is that all of the robotic hysterectomies were performed by one of three certified surgeons. But it should also be noted that all surgeons were experienced in performing both types of robotic hysterectomies. Performing different intra-corporal vaginal cuff closure techniques using different materials likely had some effects on operative times and surgical outcomes. One should accept that surgical skills are generally enhanced through accumulated experience. Because the three-armed approach was performed in the early years of our institution's history, the demonstrated longer operation times and longer hospitalization periods for the three-armed approach should logically be attributed to the surgical expertise levels of both the surgeons and our department rather than the superiority of the twoarmed approach. On the other hand, we must stress that in this study, an established robotic hysterectomy approach was compared to the two-armed approach, a technique that is under development and is not widely accepted around the world. Because of these identified biases, the results of this study must be accepted with caution until they are confirmed by future prospective studies.

\section{Conclusions}

Our data demonstrated similar surgical outcomes and complication rates when either of the approaches identified in the study was chosen for robotic hysterectomy. Reduction of one arm may prove valuable as an effective cost-minimizing strategy for robotic simple hysterectomy that potentially offers additional benefits, such as reduced vaginal dehiscence rates and increased esthetic benefits for patients. The results of this study should be verified through further prospective studies with a larger sample size. Additionally, the results have demon- strated that this approach has the potential to be widely accepted and preferred by medical communities where cost reduction is one of the primary determinants of surgery type.

\section{Conflict of interest}

The authors declare no conflict of interest.

\section{References}

1. Agency for Healthcare Research and Quality. Health Services Research on Hysterectomy and Alternatives. Available at: http:// www.ahrq.gov/research/hysterec.htm. Accessed 8/14/2010.

2. Advincula AP, Wang K. Evolving role and current state of robotics in minimally invasive gynecologic surgery. J Minim Invasive Gynecol 2009; 16: 291-301.

3. Nieboer TE, Johnson N, Lethaby A, et al. Surgical approach to hysterectomy for benign gynecological disease. Cochrane Database Syst Rev 2009; 3: CD003677.

4. Wright JD, Herzog TJ, Tsui J, et al. Nationwide trends in performance of inpatient hysterectomy in the United States. Obstet Gynecol 2013; 122: 233-41.

5. Jacoby VL, Autry A, Jacobson G, et al. Nationwide use of laparoscopic hysterectomy compared with abdominal and vaginal approaches. Obstet Gynecol 2009; 114: 1041-8.

6. Liu H, Lu D, Wang L, et al. Robotic surgery for benign gynecological disease. Cochrane Database Syst Rev 2012; 2: CD008978.

7. Chen CCG, Falcone T. Robotic gynecologic surgery: past, present, and future. Clin Obst Gynecol 2009; 52: 335-43.

8. Lenihan JP, Kovanda C, Seshadri-Kreaden U. What is the learning curve for robotic assisted gynecologic surgery? J Minim Invasive Gynecol 2008; 15: 589-94.

9. Schreuder HWR, Verheijen RHM. Robotic surgery. BJOG 2009; 116: 198-213.

10. Sarlos D, Kots L, Stevanovic N, et al. Robotic compared with conventional laparoscopic hysterectomy: a randomized controlled trial. Obstet Gynecol 2012; 120: 604-11.

11. Parasio MF, Jelovsek JE, Frick A, et al. Laparoscopic compared with robotic sacrocolpopexy for vaginal prolapse: a randomized controlled trial. Obstet Gynecol 2011; 118: 1005-13.

12. Parasio MF, Ridgeway B, Park AJ, et al. A randomized trial comparing conventional and robotically assisted total laparoscopic hysterectomy. Am J Obstet Gynecol 2013; 208: 368.el-e7.

13. Anger JT, Mueller ER, Tarnay C, et al. Robotic compared with laparoscopic sacrocolpopexy: a randomized controlled trial. Obstet Gynecol 2014; 123: 5-12.

14. Rosero EB, Kho KA, Joshi GP, et al. Comparison of robotic and laparoscopic hysterectomy for benign gynecologic disease. Obstet Gynecol 2013; 122: 778-86.

15. Wright JD, Ananth CV, Lewin SN, et al. Robotically assisted vS laparoscopic hysterectomy among women with benign gynecologic disease. JAMA 2013; 309: 689-98.

16. Wright JD, Burke WM, Wilde ET, et al. Comparative effectiveness of robotic versus laparoscopic hysterectomy for endometrial cancer. J Clin Oncol 2012; 30: 783-91. 
17. Sarlos D, Kots LA. Robotic versus laparoscopic hysterectomy: a review of recent comparative studies. Curr Opin Obstet Gynecol 2011; 23: 283-8.

18. Barbash GI, Glied SA. New technology and health care costs - the case of robot-assisted surgery. N Engl J Med 2010; 363: 701-4.

19. Wright KN, Jonsdottir GM, Jorgensen S, et al. Costs and outcomes of abdominal, vaginal, laparoscopic and robotic hysterectomies. JSLS 2012; 16: 519-24.

20. Woelk JL, Borah BJ, Trabuco EC, et al. Cost differences among robotic, vaginal, and abdominal hysterectomy. Obstet Gynecol 2014; 123: 255-62.

21. Uccella S, Ceccaroni M, Cromi A, et al. Vaginal cuff dehiscence in a series of 12,398 hysterectomies: effect of different types of colpotomy and vaginal closure. Obstet Gynecol 2012; 120: 516-23.

22. Uccella S, Ghezzi F, Mariani A, et al. Vaginal cuff closure after minimally invasive hysterectomy: our experience and systemic review of the literature. Am J Obstet Gynecol 2011; 205: 119 e1-12.

Received: 23.03.2017, accepted: 26.04.2017. 